Document downloaded from:

http://hdl.handle.net/10251/62300

This paper must be cited as:

Gisbert Paya, J.; Ibañez García, F.; Bonet Aracil, MA.; Monllorpérez, P.; Díaz-García, P.; Montava Seguí, IJ. (2009). Increasing hydration of the epidermis by microcapsules in sterilized products. Journal of Applied Polymer Science. 113(4):2282-2286. doi:10.1002/app.30210.

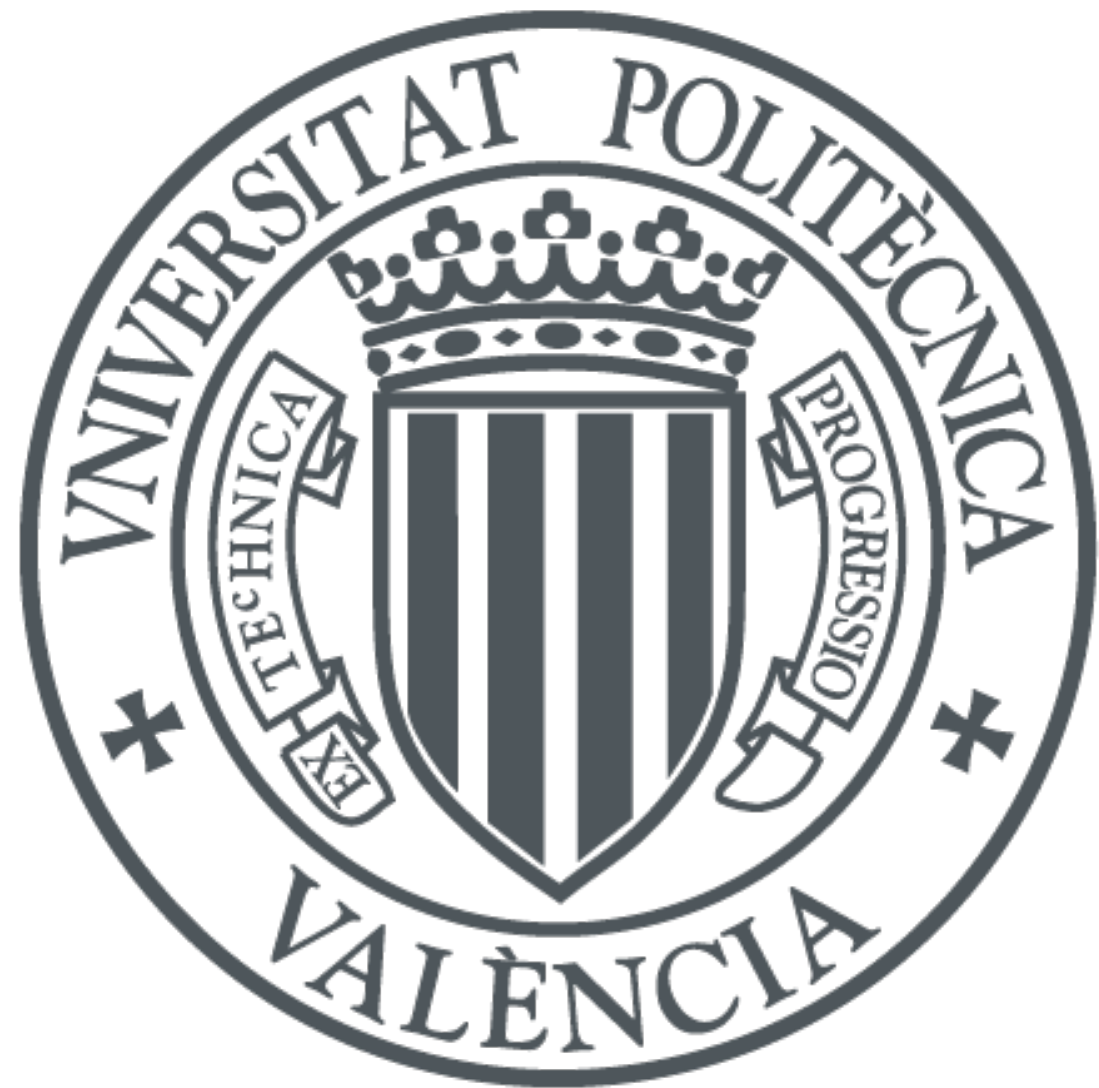

The final publication is available at

http://dx.doi.org/10.1002/app.30210

Copyright Wiley

Additional Information

This is the accepted version of the following article: Gisbert, J., Ibañez, F., Bonet, M., Monllor, P., Díaz, P. and Montava, I. (2009), Increasing hydration of the epidermis by microcapsules in sterilized products. J. Appl. Polym. Sci., 113: 2282-2286, which has been published in final form at http://dx.doi.org/10.1002/app.30210. 


\title{
INCREASING HYDRATION OF THE EPIDERMIS BY MICROCAPSULES IN STERILIZED PRODUCTS
}

\author{
J. GISBERT ${ }^{1}$, F. IBAÑEZ ${ }^{1}$, M. BONET ${ }^{\star 2}$, P.MONLLOR $^{2}$, P. DÍAZ ${ }^{2}$, I.MONTAVA $^{2}$ \\ ${ }^{1}$ Innovatec SC, S.L. Juan Carlos I n. 22-24. 03440 lbi. Spain \\ ${ }^{2}$ Universidad Politécnica de Valencia. Plaza Ferrándiz y Carbonell s/n 03801. Alcoy. Spain.
}

Correspondence: Maria Angeles Bonet, Dept. de Ingeniería Textil y Papelera, Plaza

Ferrandiz y Carbonell s/n, 03801 Alcoy, Alicante, Spain. Fax: +34 96.652.84.70.

Email:maboar@txp.upv.es

\section{KEY WORDS}

Radiation

Microencapsulation

Electron beam irradiation

Electron microscopy

\section{ABSTRACT}

Some non-serious skin infections can be treated by hydration and antibacterial control. Microcapsules containing aloe-chitin are often used to treat this kind of problem. Microcapsules were applied to cotton fabrics by padding and sleeves were prepared. A hypoallergenic test was applied to the microcapsule emulsion and hydration of the epidermis was evaluated by capacitance methods. The fabric was sterilized by electron beam treatment to satisfy the antibacterial requisite. The results showed that the aloe is transferred from the fabric to the skin, increasing the level of skin hydration. The electron beam method was also shown to be effective for bacteria and fungi and had no effect on the microcapsule properties. It can therefore be confirmed that electron beam sterilization has no harmful effects on the type of microcapsule used in this study. 


\section{INTRODUCTION}

Skin represents one part of the body that is widely shown to other people. The skin's appearance reflects the health of a person, and hydration level is one of its most important parameters. Skin moisturizers are becoming increasingly popular as women and men continue to grow in awareness of the importance of the health of their skin. [1]

Some studies showed than more than $70 \%$ of patients suffering from atopic dermatitis considered it can affect their life as it implies an alteration in their quality of life. Dermatological diseases do not only affect the life of the child but also the life of his/her parents and possibly the whole family [2] .

Treatment is often challenging, because no well-studied or approved systemic options exist [3]. Systemic options are used for severe situations, and topic treatments are prescript for non severe or in order to prevent it to appear. These infections can sometimes be treated by hydrating the skin and ensuring that the zone is free of harmful microorganisms [4-6]. Fabrics can therefore be useful if they can keep skin clean of microorganisms and improve hydration of the dermis.

To keep skin clean of microorganisms chitin is a suitable product to be applied on textiles. Chitin, poly- $(1,4)$-2-acetoamido-2-deoxy- $\beta$-D-glucose, is the second most abundant natural polymer. Its chemical structure is similar to that of cellulose, differing only in the second carbon position, where the hydroxy groups are replaced by an amino acetyl group. Chitosan is the deacetylated form of chitin, i.e. poly-(1,4)-2-amido-2-deoxy- $\beta$-D-glucose. Chitosan has been found to be capable of inhibiting microbial growth [7-8]. The antimicrobial mechanism is not clear but it is generally accepted that the primary amine groups provide positive charges which interact with negatively charged residues on the surface of microbes. Such interaction causes extensive changes in the cell surface and cell permeability, leading to leakage of intracellular substances. 
Chitosan has been used as a shell for microencapsulated products [9-10] and has also been used in textiles as a skin moisturiser with, for example, an aloe core.

The determination of skin hydration in vivo is often made indirectly by measuring some property of the skin that is correlated with water content [11]. Two methods commonly used to collect skin moisture information are based on electrical properties: capacitance and alternating current conductivity [12]. The skin hydration level can be determined by the corneometric method, as some studies have demonstrated [12-15]. The corneometric method is based on the measurement of the capacitance in a dielectric. When a change occurs in the hydration of the dermis, the dielectric constant changes and thus causes changes in capacitance.

Certain methods are normally used for sterilization: autoclave, ethylene oxide gas, and radiation. Autoclave needs a high temperature to kill bacteria and other microorganisms. Ethylene oxide gas causes surface sterilization only and can leave toxic residues. Radiation is usually by gamma rays or electron beam, the possibility of entering certain substrates is very high and penetration is superior to that of UV radiation. The use of radiation processing has become widespread in recent years. It is considered as a safe, reliable, and economical technique capable of being used in various fields of industrial production and scientific research and is an environment-friendly alternative to other industrial techniques [16]. Radiation is used in different forms, but most attention is given to those able to pass through matter and that can be considered as ionizing radiation [17]. Physico-chemical techniques such as irradiation by accelerated electron beams, neutrons or gamma radiation possess a high potential for modifying textile fibres and other materials [18].

Fabrics used in medical applications need to be sterilized. In recent years new functions have been conferred on textiles by means of incorporating microcapsules. The sterilization process, when necessary, should not be harmful to microcapsules. 
The aim of this work was to develop a product to increase the hydration level of the epidermis and that does not allow microorganisms to grown up. With this aim, fabrics were prepared and tested to check their hypoallergenic and hydration effects on skin. To ensure no microorganisms are on fabric surface, sterilization was carried out by radiation process.

\section{2.- MATERIALS AND METHODS}

\section{1.- Fabric}

Aloe-cored, chitin-walled Color-Center Microcapsules were applied to cotton fabric as purchased. Chitin is used in wall because it is insoluble, and microcapsules should be broken because of rubbing when fabric is used and then aloe gets in contact with skin. If aloe is not protected with insoluble agents, it will be lost in for example washing process, what would reduce their presence and hydration effect. Fabric was an optical blanched cotton interlock knitting fabric about $200 \mathrm{~g} / \mathrm{m}^{2}$. Padding was made with a TEPA foulard, in conditions that allowed a pick up of $90 \%$. Bath composition was $30 \mathrm{~g} / \mathrm{L}$ of microcapsules emulsion and with a $20 \mathrm{~g} / \mathrm{L}$ acrylic binder.

\section{2.- Hypoallergenic Tests.}

Subsequent tests were carried out to check the effect of the treated fabric. The first involved hypoallergenicity and the second the skin-hydrating effect

In order to test skin reaction to the aloe-chitin compound, a number of patches were applied to the subjects' skin. Patches were occlusive aluminium (50 $\mathrm{mm}^{2}$ ), Finn chamber standard, to which $20 \mathrm{ml}$ of the product under test had been added. Patches were applied 9 times in three consecutive weeks. One control test included water, as proposed by Marzulli and Maibach [18]. 
Some patches were left on the skin for 48 hours and the rest for 72 hours. Controls were carried out immediately after the test and after a 15-minute interval. All patients were interviewed to check for the possible influence of any other products they might have used and to detect procedural mistakes. To be considered as hypoallergenic, of the 55 persons involved in the study, 48 should not show any reaction.

\section{3.- Hydration test.}

This test was carried in two parts for each subject, one for the specific test and the other as a placebo. Some cotton interlock fabric sleeves contained microcapsules and the rest were placebos. Twenty five subjects suffering from dry skin were selected. They used the sleeves with microcapsules on one side and the others on the other side, each subject didn't know which was each one. Dielectric properties are directly related with skin hydration, and they were determined by capacitance measurements. Hydration was determined by the capacitance test after different periods of time (2, 4 and 6 hours) following a previously published protocol [19-22]. Measurement at 0 hours was established as base line. This test was performed by EVICHispania laboratories.

The results of the corneometric test are expressed arithmetically, based on the average value obtained from all the test subjects, seen in expression 1.

$$
\text { Hydration effect }(H E)=T_{x} / T_{0}(1)
$$

Where: $\mathrm{HE}$ is the hydration effect, and $\mathrm{T}_{0}$ indicates the corneometric index just before the test. $T_{x}$ is related to the corneometric value after 2, 4 and 6 hours, as defined in the procedure.

The real hydration effect, or increased hydration effect (IHE) was obtained as the difference between the results from the placebo zone and the treated zone, as seen in expression 2.

$$
\mathrm{IHE}=(\mathrm{HE})_{\text {microcapsules zone }}-(\mathrm{HE})_{\text {placebo zone }}
$$


Where: $(\mathrm{HE})_{\text {microcapsules zone }}$ corresponds to the $\mathrm{HE}$ in the zone treated with fabrics not containing microcapsules, and $(\mathrm{HE})_{\text {placebo zone }}$ represents $\mathrm{HE}$ in the control zone, in which some fabrics without microcapsule products were tested.

\section{4.- Sterilization process.}

Sleeves were treated by electron beam in order to destroy all microbial presence on the fabric. To avoid re-contamination while waiting to be used, the fabric was thermally enclosed in polyethylenglicol. The treatment was carried out at the recommended IONMED intensity.

\section{5.- Microbiological and antibacterial analysis.}

ASTM E2149-01 was tested to evaluate the resistance of non-leaching antimicrobial-treated specimens to the growth of microbes under dynamic contact conditions.

Microbiological analysis was carried out with aerobic bacteria at $35^{\circ} \mathrm{C}$ on the total amount of fungus.

In both tests, samples were checked before and after sterilization treatment by AITEX, a Spanish textile technology institute.

2.6.- Scanning Electron Mycrocspy (SEM).

A JEOL JSM-6300 scanning electron microscope (SEM) was used for surface observation. Each sample was fixed on a standard sample holder and sputter coated with gold. It was then examined with by the SEM with suitable acceleration voltage $(10 \mathrm{kV})$ and magnification. 


\section{3.- RESULTS}

\section{1.- Hypoallergenic test.}

The hypoallergenic test was performed in order to demonstrate that microcapsule emulsion based on aloe-chitin added to fabrics posed no danger to skin. A dermatologist evaluated the effect on the skin in four categories: very good, good, medium and bad.

The test was performed on 55 persons. The tests could be classed as successful if at least 48 persons showed no reaction to the product. In no case was a reaction found, which means that none of the subjects was sensitive to the patches used. If a reaction were to occur with patches containing $20 \mathrm{ml}$ of aloe-chitin microcapsule emulsion, it would imply a reaction caused by the tested product.

As none of the subjects showed any kind of reaction, thus allows as concluding that the aloe-chitin compound applied to fabrics was hypoallergenic.

\section{2.- Hydration test.}

Table 1, shows the results obtained from the test. Different behaviour can be observed in hydration after 2, 4 and 6 hours. Hydration increases quite quickly after two hours, but 4 hours later (6-hour test) the value is not much higher, and seems to remain constant. For this reason the test period was limited to 6 hours. 
The result after 2 hours shows an increase in hydration, also, but not so significantly, after 4 hours, and it can be considered stabilised after 6 hours. We should perhaps indicate here that clothes are usually worn for longer than 6 hours. It can therefore be affirmed that the effect can produce an increase in skin hydration of about $11 \%$. This quantity is quite important and we can consider it significative to prevent illness, as some doctors we asked for corroborated it. We think it can be increased by means of introducing more microcapsules to fabric.

In order to determine whether the effect was produced by the aloe included in the microcapsules, a weight control was carried out. If the skin has been hydrated by the aloe, it can be assumed that the microcapsules have been broken and that their contents (aloe) have permeated into the skin, involving a weight loss in the fabric. Before weighting samples all of them were in a room with $20^{\circ} \mathrm{C}$ and $65 \%$ of relative moisture for at least 24 hours in order to get them conditioned.

The results indicated a weight loss after 6 hours. To rule out the possibility of weight loss through loss of fibre mass, the placebo fabrics were also weighed after 6 hours. The results can be seen in Table 2, $p$ value is lower than 0.001 for every sample.

\section{Insert table 2 about here.}

It can be observed that weight loss is greater in fabrics containing microcapsules than in placebo fabrics. To confirm the significance of the result, statistical analyses by Student $t$ test were carried out which showed that the weight difference was significant. It can therefore be concluded that the increase in skin hydration was due to the effect of the aloe contained in the microcapsules.

To check it is due because microcapsules have been broken and aloe disappear from fabric we made SEM analysis which can be seen in figure 2. 
In figure 1 we can observe some chitin wall that show not sphere shape, because it has been broken, consequently aloe has gone out and get in contact with skin.

\section{4.- Sterilization process.}

Fabrics were sterilised by electron beam and were subsequently analyzed to determine the effects. The fabrics padded with microcapsules were evaluated by SEM. Figure 2 shows the presence of microcapsules in the fabric before and after sterilization.

\section{Insert figure 2 about here}

It can be observed that there is no appreciable difference in the aspect of the microcapsules before and after the fabric has been sterilized. If core product had been lost because of the treatment, spheres would be deflated. However, there is no evidence to show whether the electron beam has reduced the antibacterial effectiveness of the chitin wall.

The assessment of antibacterial activity of Escherichia coli on the textile material with microcapsules was about 57,0 \% at 0 hours and about 60,3\% 1 hour later. No significant differences were found when the test was performed with sterilized fabrics.

On the other hand, we studied the convenience of sterilizing the fabric. We tested fungus and aerobic bacteria of one fabric without being sterilized and we saw results showed in table 3 . We did find a big difference due to the sterilization process when the results of the microbiological analysis were studied. These are expressed as the number of units developed on cells over dried weight (Table 3). 


\section{Insert table 3 about here}

These results provide evidence that the electron beam treatment reduced the microbial population on the treated fabric and, furthermore, had no harmful effect on the action of the microcapsules.

As we said before, it is necessary to treat damaged skin with hydrating products, and to prevent it from microorganisms. This test shows that if the textile product is sterilized it will not have microorganisms and is ready to be used directly on affected skin.

\section{4.- CONCLUSIONS}

As a result of the hypoallergenic test, we could confirm that none of the subjects had shown any kind of reaction. It can therefore be concluded that the aloechitin microcapsule emulsion applied to the fabric was hypoallergenic. No other similar products have so far been tested in this way. Microcapsules product will hydrate skin because chitin wall will be broken because of rubbing while textile is used and then aloe is in contact with skin.

Epidermal hydration was determined as the difference between hydration in the treated zone with respect to that covered by the placebo. We confirmed that it increased by about $11 \%$ after 6 hours of application of the fabric. Hydration was seen to rise faster at the beginning and had practically stabilized after about 4 hours. A weight test was then performed to verify that the rise was due to the aloe from the fabric permeating the skin. The time needed to stabilize the hydration level can be influenced by the concentration of microcapsules in the fabric, but in this study the microcapsule concentration was constant. Further work will be carried out to determine hydration rate at higher concentrations. The SEM analysis shows that there are no apparent effects on microcapsules due to the sterilization process. The study of antimicrobial effectiveness and antibacterial activity confirmed that the microcapsules had lost none of their properties. The microbiological analysis also confirms the effectiveness of the sterilization process in reducing total fungi population and aerobic bacteria. 


\section{REFERENCES}

1 Dal Farra, C., Antipolis, S., Oberto, G., Peyronel, D., Domloge, N. “In vivo studies of the effects of a new rice extract on skin hydration andbarrier function" Journal of the American Academy of Dermatology Volume 58, Issue 2 (2). 203. 2008.

2 Taieb, C., Fabre, P., Cedex, B., Martinsic M. A. "Atopic dermatitis and thermal therapy: Evaluation of medical service". Journal of the American Academy of Dermatology Volume 58, Issue 2 (2). p110. 2008.

3 Rouse, C; Siegfried, E ."Methotrexate for atopic dermatitis in children" Journal of the American Academy of Dermatology Volume 58, Issue 2 (2).,p608. 2008.

4 Alekseev, SI., Szabo, I., Ziskin MC. Millimeter wave reflectivity used for measurement of skin hydration with different moisturizers..Skin Res Technol. Nov;14(4):390-396. 2008.

5 Fisher AA. Contact dermatitis, 3rd edn. Philadelphia: Lea and Febiger,: 801-842.1988.

6 Kligman AM. Hydration injury to human skin. In: Elsner P, Berardesca E, Maibach $\mathrm{HI}$, eds. Bioengineering of the skin: water and the stratum corneum. Boca Raton: CRC Press, 251-255. 1994.

7 Gao, Y. and Granston, R..Recent advantages in antimicrobial treatments of textiles. Textile Research Journal, Textil Research Journal. 78(1). pp 60-72. 2008.

8 Lim, S. H., and Hudson, S. M., Review of Chitosan and its Derivatives as Antimicrobial Agents and Their uses as Textile Chemicals, J. Macromol. Sci. Polymer Rev., 43, 223-269. 2003.

9 S. Ozbas, C. Aral, L. Kabasakal, M. uysal, J. Akbuğa. Co-encapsulation of two plasmids in chitosan as non-viral gene delivery vehicle. J. Pharm. Pharmaceut. Sci. 6(1). Pp 27-23. 2003.

10 Bartkowiak, A., Hunkeler D. Carrageenan-oligochitosan microcapsules: optimization of the formation process Colloids and Surfaces $B$ : 
Biointerfaces 21 285-298. 2001.

11 Attas, A.;Posthumus, M.;Schattka, T.; Sowa, B.; Mantsch, M.; Zhang, H.; Long-wavelength near-infrared spectroscopic imaging for in-vivo skin hydration measurements. Vibrational Spectroscopy 28.1. 37-43.2002.

12 Bettinger, J.; Gloor, M.; Vollert, A.; Kleesz, P.; Fluhr J.; Gehring, W. Comparison of different non-invasive test methods with respect to the effect of different moisturizers on skin. Skin Res. Technol. 5 p. 2127.1999.

13 Rodrigues, L.M.; Pinto, P.C. Analysis of the influence of hidration of the epidermis on the biomechanical behaviour of in vivo skin. Ars. Pharmaceutica, 45:1; 59-71, 2004.

14 Pellacani, G., Seidenari, S."Water sorption- Desorption Test and moisture Accumulation Test for Functional Assesment of Atopic Skin in Children". Acta Demato-Venereologica. Vol 81.n2,. pp 100-103. 2001.

15 Yosipoviitch, G., Maayan-Metzger, A., Merlob, P., Sirota, L.. "Skin Barrier properties in different body areas in Neonates". Pediatrics. Vol. 106. n1. pp 105-108. 2000.

16 Auditore, L., Barnà, R.C., de Pasquale, D., Italiano, A., Trifirò, A., Trimarchi, M.. "Pulsed 5 Mev standing wave electron linac for radiation processing". The american Physical Society. Physical review special topics. Accelarators and beams. Vol 7. 030101. Pp - 030101-1 - 0301015. 2004.

17 Landete-Ruiz, M.D., Martín Martinez, J.M.. Surface Modification of EVA copolymer by UV treatment. Internacional Journal of Adhesión and Adhesives, 25 (2). pp.139 - 145. 2005.

18 Marzulli, F.N., Maibach, H.I., Contact allergy: predictive testing in man. Contact Dermatitis, 2, pp 1-17. 1976.

19 Berardecasa, E., EEMCO guidance for the assessment of stratum corneum hydration: electrical methods. Skin research and technology, 3, pp 126-132. 1997

20 Clarys, P., Barel, A.O. In vitro calibration of the capacitance method (Corneomether CM $825 \AA$ ) and conductance method (Skicon $200 \AA$ ) for the evaluation of the hydration state of the skin. Skin research and 
technology, 3, pp 107-113. 1997.

21 Clarys, P., Barel, A.O., Gabard, B. Non invasive electrical measurements for the evaluation of the hydration state of the skin: comparison between three conventional instruments- the Cornemometer $\AA$, the Skicon $\AA$ and the Nova DPM $\AA$. Skin research and technology, 5 , pp 14-20. 1999.

22 Fluhr, J. W. and col. Comparative study of five instruments measuring stratum corneum hydration (Cornemometer $\mathrm{Cm} 820$ and $\mathrm{CM} 825 \AA$, the Skicon $\AA$, the Nova DPM $9003 \AA$, Dermalab $\AA)$ Part II In vivo. Skin research and technology, 5, pp 171-178. 1999. 


\section{FIGURE CAPTIONS}

Figure 1.- Broken wall of microcapsules after being tested the fabrics.

Figure 2.- SEM images of cotton interlock fabric treated with aloe-chitin microcapsules. a)

Before being sterilized. B) After being sterilized. 
FIGURES

Figure 1

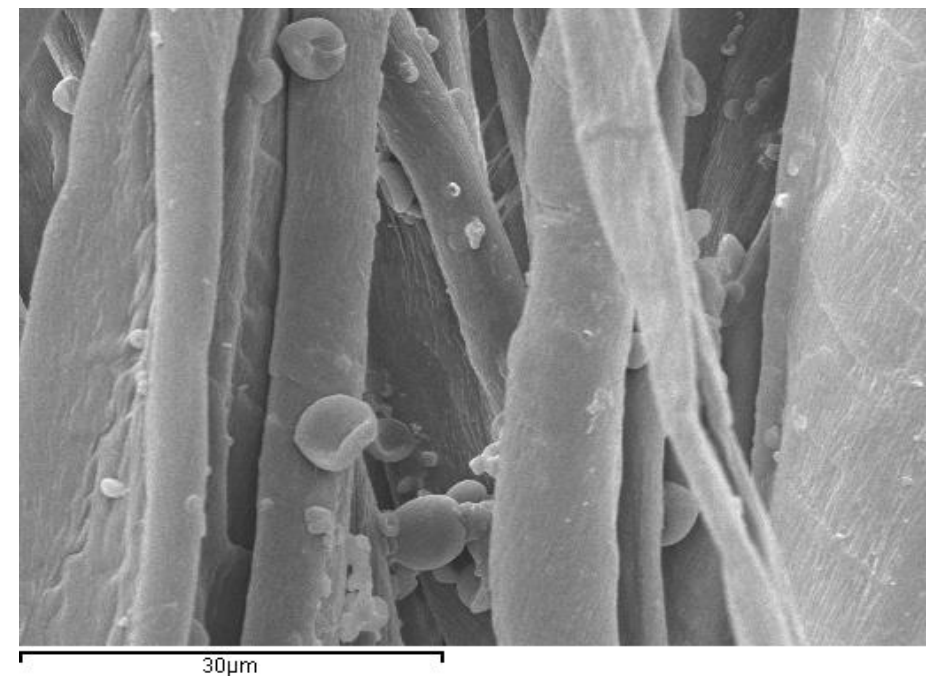


Figure 2a

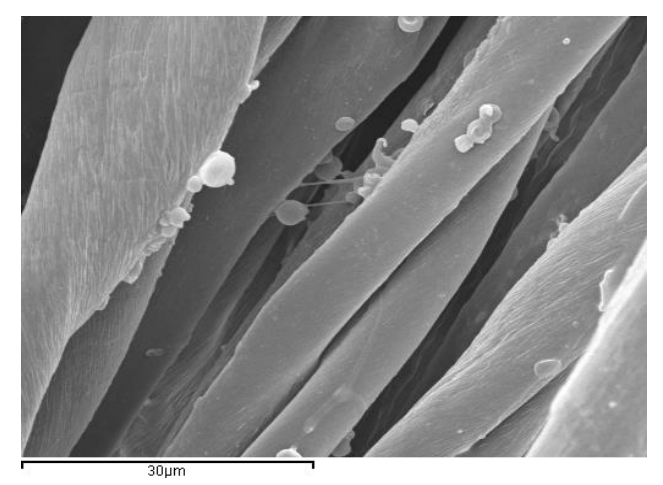


Figure $2 b$

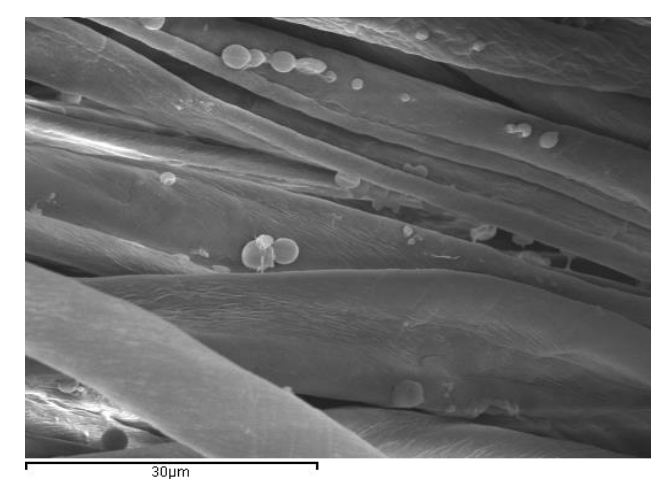




\section{TABLES}

Table 1.- Increase in skin hydration by corneometric measurement, and standard deviation.

\begin{tabular}{cccc}
\hline & \multicolumn{3}{c}{ Time (hours) } \\
\hline $\begin{array}{c}\text { Hydration } \\
\text { Increase }\end{array}$ & $7.1 \%$ & $\mathbf{4 h}$ & $\mathbf{6} \mathbf{h}$ \\
\hline $\begin{array}{l}\text { Standard } \\
\text { deviation }\end{array}$ & $1.7 \%$ & $9.9 \%$ & $10.7 \%$ \\
\hline
\end{tabular}


Table 2.- Weight lost in fabrics.

\begin{tabular}{ccccccc}
\hline & \multicolumn{7}{c}{ Weight (g) } \\
\hline & \multicolumn{7}{c}{ Fabric with microcapsules } & \multicolumn{4}{c}{ Placebo fabric } \\
\hline & T0 & T6 & T0-T6 & T0 & T6 & T0-T6 \\
\hline Average & 48.004 & 47.788 & -0.216 & 37.048 & 36.945 & -0.103 \\
\hline $\begin{array}{l}\text { Standard } \\
\text { derivation }\end{array}$ & 0.445 & 0.445 & 0.082 & 0.204 & 0.189 & 0.061 \\
\hline
\end{tabular}


Table 3.- Microbiological analysis.

BEFORE

STERILIZATION

AFTER STERILIZATION

PARAMETER

ufc/g over dried weight. ufc/g over dried weight.

\begin{tabular}{ccc}
\hline Fungus & 12 & $<4$ \\
\hline $\begin{array}{c}\text { Aerobic bacteria } \\
(35 \circ)\end{array}$ & 184 & $<4$ \\
\hline
\end{tabular}

